\title{
Therapeutic Mammoplasty in Management of Breast Cancer: A Prospective Clinical Study
}

\author{
Ahmed Mohamed Farahat, Amr Abdel Meguid Attia, Mohamed Aly M. El Shafei El Zawahry, \\ Mohamed H. Zedan, Hussein Osama Soliman*
}

National Cancer Institute, Cairo University, Giza, Egypt

Email: ^soliman@daad-alumni.de

How to cite this paper: Attia, A.A.M., Farahat, A.M., El Shafei El Zawahry, M.A.M., Zedan, M.H. and Soliman, H.O. (2017) Therapeutic Mammoplasty in Management of Breast Cancer: A Prospective Clinical Study. Advances in Breast Cancer Research, 6, 107-116.

https://doi.org/10.4236/abcr.2017.64010

Received: March 6, 2017

Accepted: September 17, 2017

Published: September 20, 2017

Copyright $\odot 2017$ by authors and Scientific Research Publishing Inc. This work is licensed under the Creative Commons Attribution International License (CC BY 4.0).

http://creativecommons.org/licenses/by/4.0/

\begin{abstract}
Background: The introduction of oncoplasticbreast surgery, with a concomitant contralateral symmetrization procedure, allows the surgical oncologist the freedom to perform wider excisions, with more favorable cosmetic outcomes. Objective: To assess the feasibility of bilateral breast reduction for the management of breast cancer, and to determine the most suitable surgical technique of therapeutic mammoplasty for each patient. And to evaluate the cosmetic outcome during the postoperative period. Methods: This study was from June 2014 until June 2016, and included 86 female patients with breast cancer; divided in 3 groups; group A; had bilateral superior pedicle reduction mammoplasty, group B; had bilateral inferior pedicle reduction mammoplasty, group C; had bilateral batwing mastopexy. 1 month postoperative, patients were asked to answer a 5-scale questionnaire evaluating their own cosmetic outcome. Criteria they were asked to evaluate were: symmetry, shape and volume, projection, correction of ptosis, visibility of the scars and overall satisfaction. A similar questionnaire was answered by a panel made up of a surgeon and breast-care nurse after seeing the pre- and post-operative photos of the patients in question. Results: Group A had a mean tumor size of $2.7 \pm 0.69$ $\mathrm{cm}$, least resected margins $1.2-4.3 \mathrm{~cm}$, Group B had a mean tumor size of 3.2 $\pm 1.19 \mathrm{~cm}$, least resected margins $0.7-3.8 \mathrm{~cm}$, Group $\mathrm{C}$ had a mean tumor size of $3.4 \pm 1.12 \mathrm{~cm}$, least resected margins $1.7-5.2 \mathrm{~cm}$. Cosmetic results fared high above average. Group $\mathrm{C}$ fared better in overall satisfaction but Group B fared better in ptosis correction, projection and symmetry. The panel answers showed difference regarding shape and volume; the only two criteria to be almost of the same result among the three groups. Conclusion: Bilateral TM has some advantages when compared to standard conventional BCS techniques without having any unfavorable effects on surgical margin confidence, local recurrence, and survival rates. So, it is a valuable addition to consider the use of such surgical techniques whenever indicated in the surgical manage-
\end{abstract}


ment of patients with breast cancer. Batwing mastopexy showed superiority in operating time, hospital stay and overall satisfaction of patients, surgeons and nurses.

\section{Keywords}

Breast Oncoplasty, Therapeutic Mammoplasty, Surgery, Oncology, Plastic, Symmetry

\section{Introduction}

Historically, the surgical treatment of breast cancer evolved into the Patey and Dyson modification of Halsted's radical mastectomy "Modified Radical Mastectomy" [1] [2], which offered oncologically equal results to Halsted's radical mastectomy, and since the 1950s was adopted as the surgical intervention of choice for patients with breast cancer.

Later on, breast-conserving surgery combined with postoperative radiotherapy was established as a safe alternative to Patey's modified radical mastectomy; in terms of survival and rate of local recurrence [3].

With tools such as neoadjuvant chemotherapy, postoperative radiation treatment and oncoplastic breast surgery, a wider range of patients can benefit from breast conservation.

\section{Aim of the Work}

- To assess the feasibility of bilateral breast reduction for the management of breast cancer.

- To determine the most suitable surgical technique of therapeutic mammoplasty for each patient.

- To evaluate the cosmetic outcome throughout different postoperative phases.

- To highlight the benefits and complications of therapeutic mammoplasty in the management of patients with breast cancer.

\section{Patients and Methods}

This was a prospective study that included 86 patients diagnosed with surgically treatable breast cancer, between June 2014 and June 2016. Patient selection was achieved through a number of inclusion and exclusion criteria, and the study was approved by the ethical committee of the National Cancer Institute, Cairo University.

\subsection{Inclusion Criteria}

Oncoplastic procedures were proposed for patients in whom breast conservative treatment is possible on oncologic grounds but in whose cases a standard resection without reconstruction would result in poor cosmesis with the following 
criteria:

1) Female patients with ages ranging from 25 to 65 years

2) Patients with early surgically treatable breast cancer (Stage I, II and IIIa)

3) Patients whose management was by upfront surgery or neo-adjuvant chemotherapy followed by surgery.

4) Patients with tumors in any quadrant of the breast

5) Patients with large/ptotic breasts (especially with brassiere size C, D or DD).

\subsection{Exclusion Criteria}

1) Patients with a contraindication to breast conservative surgery.

a) Multi-centric or wide spread disease, that can't be incorporated by local excision through a single incision that achieves adequate safety margin and satisfactory cosmetic results.

b) Women with a known genetic predisposition to breast cancer (e.g. BRCA 1 or 2 mutation).

c) Diffuse suspicious or malignant micro-calcifications.

d) Inability to attain negative surgical margins.

e) Contraindications to postoperative radiation: [4].

i) Previous chest wall or breast radiation.

ii) Radiation therapy to be scheduled during pregnancy.

iii) Significant pre-existing lung disease particularly where the diffusing capacity is reduced.

iv) Pre-existing pulmonary tuberculosis.

v) Cardiomyopathy.

vi) Connective tissue disorders (S.L.E., scleroderma, etc.) with significant vasculitis. The use of radiation may not be contraindicated with all connective tissue disorders.

2) Patients with small sized breasts, which are not suitable for reduction (with brassiere size A or B).

3) Patients who are not compliant; Non-compliant patients included those demanding mastectomy for fear of local recurrence, patients not consenting to the proposed procedure after adequate counseling, those refusing post-operative adjuvant Radiotherapy, refusal of a reduction mammoplasty of the contralateral breast as a symmetrizing procedure and patients who are not able to consent such as mentally challenged patients.

\subsection{Operative Techniques and Intra-Operative Details}

Excision of the tumor with a wide clear safety margin down to the pectoral fascia with frozen section confirmation of malignancy and negative margins was underwent by all patients, along with axillary lymph node dissection according to our standard protocol.

Immediate reconstruction of the breast was carried out using bilateral therapeutic mammoplasty as pre-planned and designed to meet the patient's re- 
quirement and a contralateral symmetrizing procedure was done concomitantly at the same setting as a marked degree of symmetry is usually anticipated.

Therapeutic Mammoplasties techniques used can be subdivided into:

- Superior pedicle technique (Group A; 10 patients).

- Inferior pedicle technique (Group B; 40 patients).

- Batwing mastopexy (Group C; 36 patients).

Depending on the site of the tumor and the area that requires resection, reduction mammoplasty was fashioned using a nipple-areola pedicle based either superiorly or inferiorly for the first two groups.

\subsubsection{Superior Pedicle Technique}

- This technique was used for wide excision of tumors in the lower pole of the breast.

- The tumor was resected with its safety margins through a wise pattern incision marked on the lower pole of the breast as shown in pictures (Figure 1).

- The same procedure was performed on the other breast in the same setting, to achieve symmetry using a new set of surgical instruments.

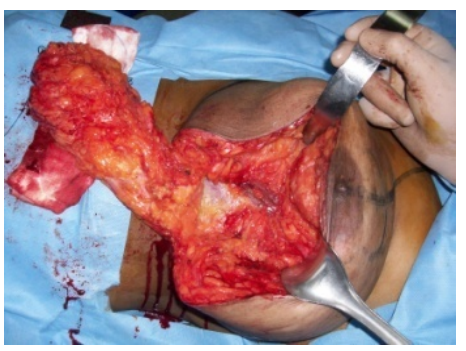

(a)

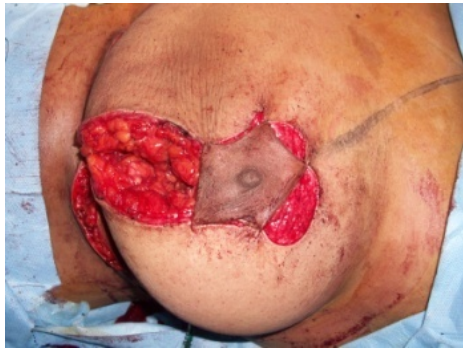

(b)

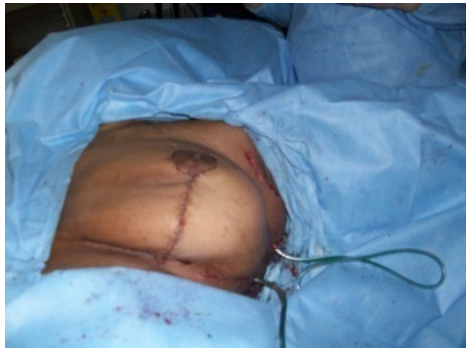

(d)

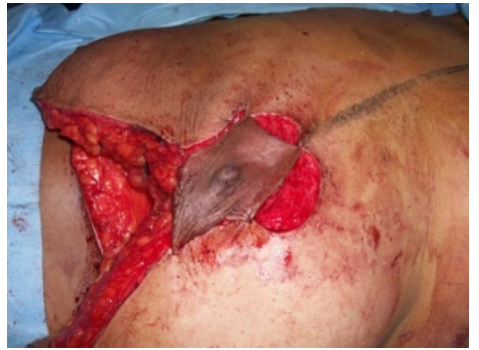

(c)

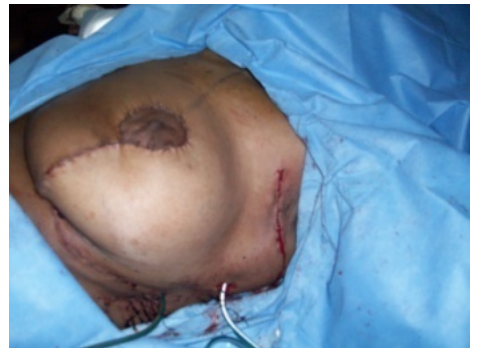

(e)

Figure 1. Pictures ((a)-(e)); surgical technique of superior pedicle mammoplasty; (a). lumoectomy; (b) \& (c) positioning of thepedicle and skin coaptation; (d) \& (e) immediate intraoperative results. 


\subsubsection{Inferior Pedicle Technique}

- This technique was best used for wide excision of tumors located above the nipple or in the upper pole of the breast.

- Mark up was done using a keyhole pattern shown in pictures (Figure 2) and the tumor or previous lumpectomy scar was placed within the area planned for resection.

- The same procedure was performed on the other breast in the same setting, to achieve symmetry using a new set of surgical instruments.

\subsubsection{Batwing Mastopexy}

- This technique was best used for wide excision of tumors located in the upper peri-areolar region, particularly when the lesions are in proximity of the skin.

- Designing of the skin incisions should be made with the patient sitting erect (Figure 3).

- The same procedure was performed on the other breast in the same setting, to achieve symmetry using a new set of surgical instruments.

\subsection{Assessment of the Cosmetic Outcome}

- Cosmetic outcome was evaluated during the early post-operative period and on follow up.

- Cosmetic outcome was evaluated by a surgeon, a breast-care nurse and the patient.

- One month after completion of their adjuvant therapy, patients were invited to answer a five-scale questionnaire evaluating their own cosmetic outcome graded as: excellent (5), good (4), fair (3), poor (2) or very poor (1). Cosmetic criteria they were asked to evaluate were symmetry, shape and volume, projection, correction of ptosis, visibility of the scars and overall satisfaction (Table 1).

- Affection of nipple sensation was assessed separately in the same questionnaire.

Table 1. Cosmetic evaluation by the surgeon, the patients and a nurse.

\begin{tabular}{ccccc}
\hline $\begin{array}{c}\text { Cosmetic } \\
\text { evaluation }\end{array}$ & Patients & Surgeon & Nurse & \multirow{2}{*}{$\boldsymbol{p \text { value* }}$} \\
\cline { 2 - 4 } Shape & Median (range) & Median (range) & Median (range) & 0.002 \\
Volume & $5(3-5)$ & $5(3-5)$ & $4(3-5)$ & 0.001 \\
Ptosis & $5(3-5)$ & $5(3-5)$ & $5(3-5)$ & 0.50 \\
Projection & $4(2-5)$ & $5(3-5)$ & $5(3-5)$ & 0.59 \\
Symmetry & $4(1-5)$ & $4(2-5)$ & $4(2-5)$ & 0.31 \\
Visibility of scar & $4(2-5)$ & $4(2-5)$ & $4(2-5)$ & 0.87 \\
Overall satisfaction & $5(2-5)$ & $4(2-5)$ & $4(2-5)$ & 0.23 \\
\hline
\end{tabular}




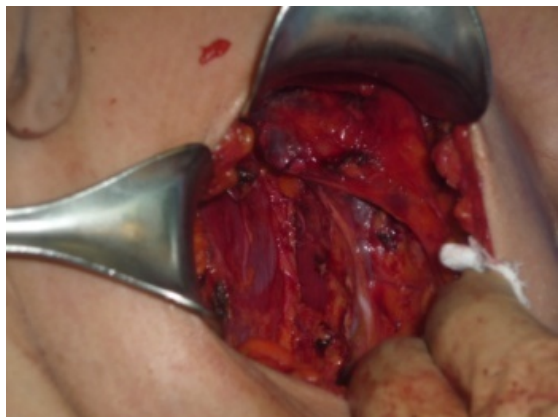

(a)

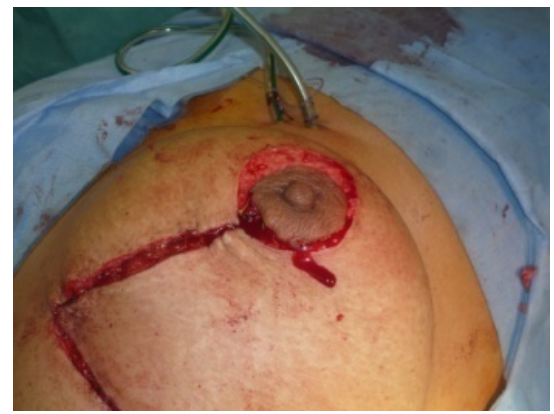

(c)

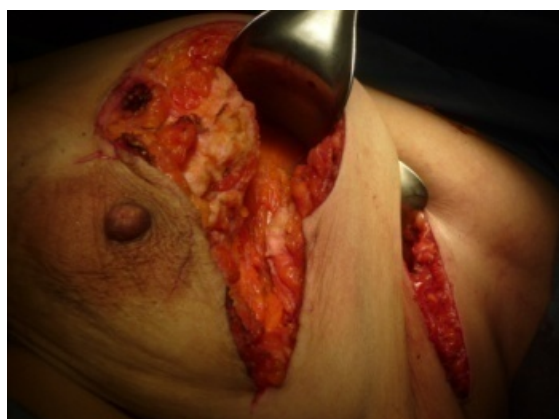

(b)

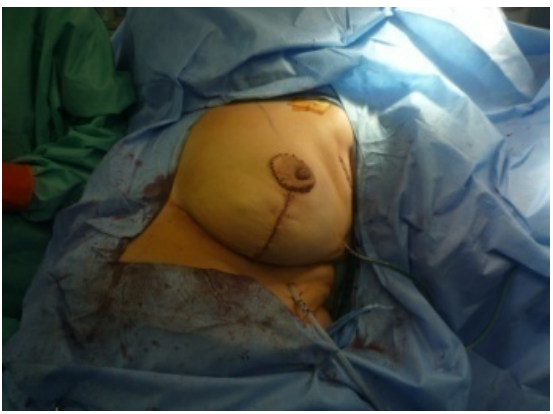

(d)

Figure 2. Pictures ((a)-(d)): surgical technique of inferior pedicle mammoplasty; (a) Lumpectomy site; (b) Skin defect; (c) Skin coaptation; (d) Immediate intra-operative result.

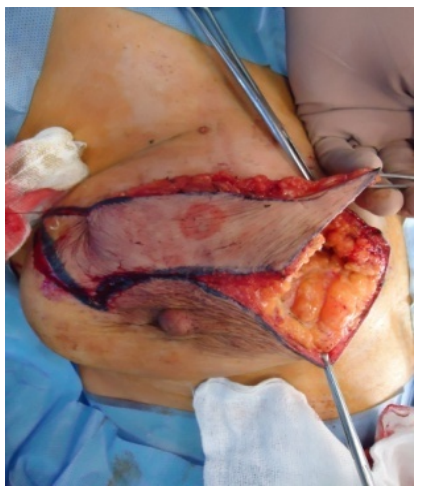

(a)

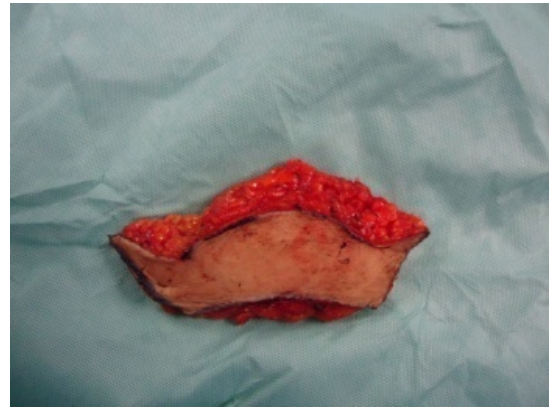

(c)

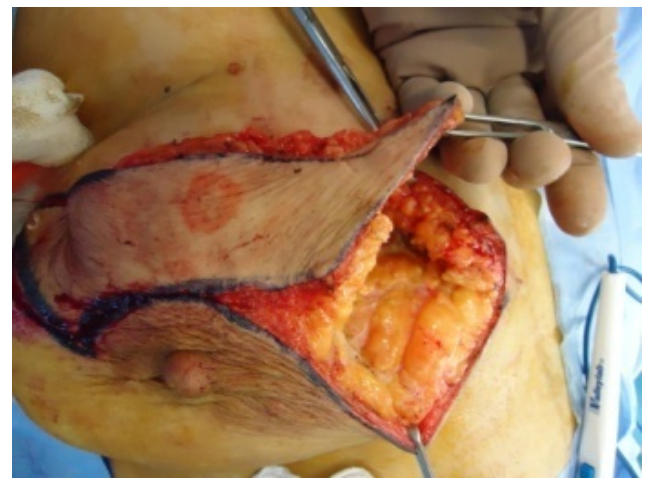

(b)

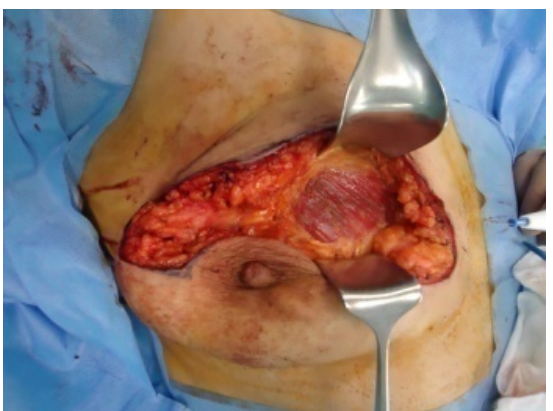

(d)

Figure 3. Pictures ((a)-(d)); surgical technique of batwing mastopexy; (a) \& (b) lumpectomy design; (c) lumpectomy specimen; (d) lumpectomy site. 
- Re-evaluation was done after completion of adjuvant chemotherapy and radiotherapy during follow up.

- Again, the panel was asked to fill in a five-scale questionnaire similar to the one handed out to the patients.

\subsection{Statistical Methodology}

SPSS version 18.0 was used for data analysis. Mean \pm standard deviation described quantitative data, with median and range for data measured on ordinal scale or do not follow normality in distribution. Kruskal Wallis ANOVA compared $>2$ independent groups and pair wise comparisons were Bonnferoni adjusted. Chi-square and Fisher Exact compared independent proportions. P value is significant at 0.05 level.

\section{Results}

\subsection{Procedure}

Group A made up of 10 patients who had bilateral superior pedicle reduction mammoplasty (11.6\%), Group B made up of 40 patients who had bilateral inferior pedicle reduction mammoplasty (46.5\%) and Group C made up of 36 patients who had bilateral batwing reduction mastopexy (41.9\%).

\subsection{Cosmetic Outcome}

The criteria being evaluated were symmetry, shape, volume, projection, correction of ptosis, visibility of the scars and overall satisfaction.

Further evaluation of the cosmetic outcome was achieved by showing a serial of pre- and post-operative pictures of each patient to a panel composed of a breast surgeon and an attending breast-care nurse.

Again, the panel was asked to fill in a five-scale questionnaire similar to the one handed out to the patients. The criteria the panel had to evaluate were symmetry, shape and volume, projection, correction of ptosis, visibility of the scars and overall satisfaction (Table 1 ).

When comparing the median of the questionnaire answers between the patients', surgeons' and nurses', regarding shape, it was found that the median for the nurses' answers was 4 (with a range of $3-5$ ). That was worse than that of the surgeons', which was 5 (with a range of $3-5$ ), and also worse than that of the patients', which was also 5 with a (range of $2-5$ ), and the difference in both was statistically significant $(p=0.002)$.

\section{Discussion}

Breast cancer is one of the commonest malignant diseases diagnosed and treated worldwide [4], and in the National Cancer Institute, Cairo University; it is the commonest malignancy: compromising $20 \%$ of all cancers and $43 \%$ of female cancers [5]. On top of that, in Egypt it has a tendency to affect a younger group of female patients. So, it might have a more devastating effect on both the physi- 
cal and psychological aspects [3] [4].

Women in Egypt tend to have bigger, more ptotic breasts, hence, the treatment of breast cancer poses a more difficult challenge to the surgeon, therefore there is a need for a surgical technique that allows the surgeon to perform oncologically safe resection, in addition to dealing with the problem of bigger more ptotic breasts and still produce the best cosmetic result possible.

The advantages of oncoplastic procedures in the treatment of breast cancer in larger and/or ptotic breasts include oncological advantages such as being able to obtain wider resection margins and conserving breasts with larger tumors but favorable tumor-breast ratio, such procedures also improve the homogenous delivery of irradiation to the breast of a woman who would have been previously deemed to undergo a mastectomy [6].

When analyzing the results of the patients' questionnaire regarding ptosis and projection, it was found that the median answer for group B was better than that for group $\mathrm{A}$ and the median answer for group $\mathrm{C}$ was better than that for group $\mathrm{A}$ and the differences were statistically significant $(p=0.5)$.

And regarding symmetry, it was found that the median answer for group B was better than that for both group A and group C and the median answer for group $\mathrm{C}$ was worse than that for both group $\mathrm{A}$ and group $\mathrm{B}$ and the differences were statistically significant $(p=0.31)$.

While regarding overall satisfaction, the median answer for group $\mathrm{C}$ was better than that for group $\mathrm{B}$ and the median answer for group $\mathrm{C}$ was also better than that for group A and the differences were statistically significant $(p=0.23)$.

This leads one to conclude that even though the patients in group B who had bilateral inferior pedicle mammoplasty was superior regarding ptosis correction and post-operative projection and symmetry, but the overall satisfaction of patients fared better in group $\mathrm{C}$ who had bilateral batwing mastopexy when compared to the other two groups.

To increase the objectivity of this study further evaluation of the cosmetic outcome was done by showing the serial of pre- and postoperative pictures of each patient to a panel composed of a breast surgeon and an attending breast-care nurse. And, the aforementioned panel was asked to fill in a five-scale questionnaire similar to the one handed out to the patients. The criteria the panel had to evaluate were symmetry, shape and volume, projection, correction of ptosis, visibility of the scars and overall satisfaction (Figure 4).

This work has been done for several years globally, but is fairly new in Egypt. The reasons behind its recent success are the wider acceptance of the procedure, especially when the patients get to see the results of the previous patients. Together with the consenting of working on the contralateral breast for better symmetrization, especially after being certain that the management of the tumor hasn't been compromised.

\section{Conclusion}

Bilateral TM has some advantages when compared to standard conventional 


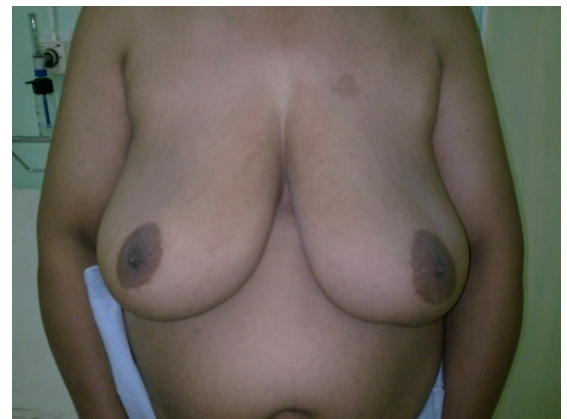

(a)

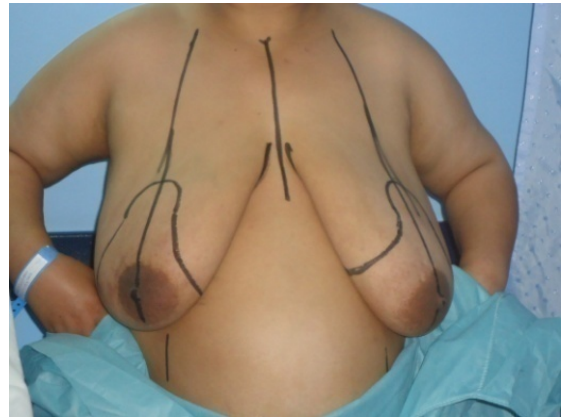

(c)

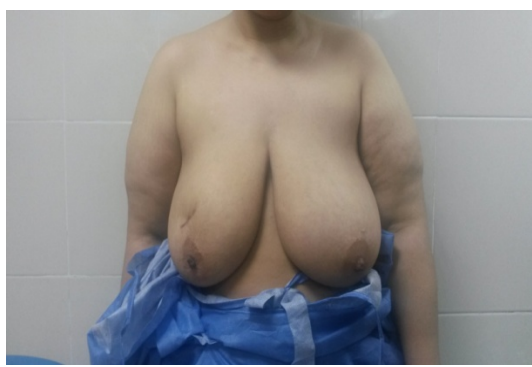

(e)

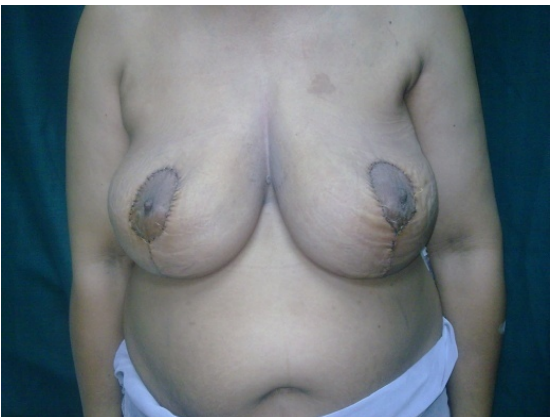

(b)

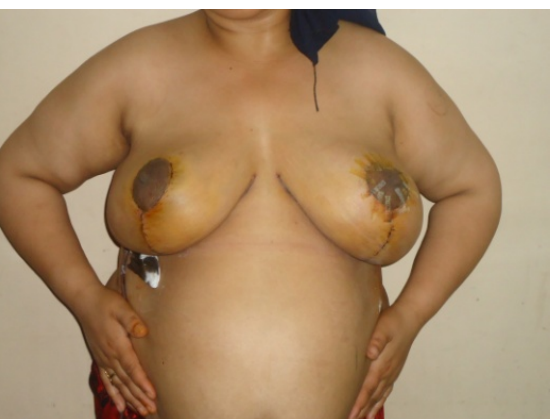

(d)

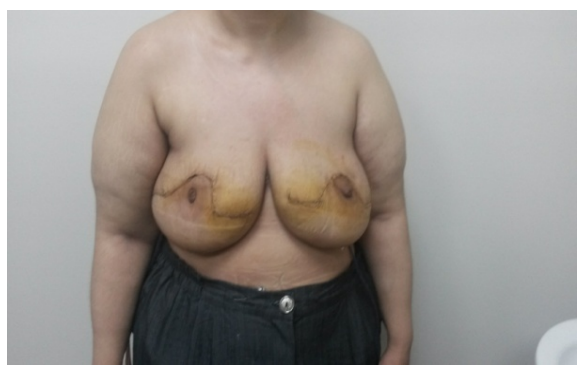

(f)

Figure 4. (a) and (b) pre and post of bilateral superior pedicle mammoplasty; (c) and (d) pre and post of bilateral inferior pedicle mammoplasty; (e) and (f) pre and post of bilateral batwing mastopexy.

BCS techniques without having any unfavorable effects on surgical margin confidence, local recurrence, and survival rates. So, it is a valuable addition to consider the use of such surgical techniques whenever indicated in the surgical management of patients with breast cancer. Batwing mastopexy showed superiority in operating time, hospital stay and overall satisfaction of patients, surgeons and nurses.

\section{References}

[1] Jatoi, I., Kaufmann, M. and Petit, J. (2006) Atlas of Breast Surgery. Springer-Verlag, Berlin Heidelberg, 1-7.

[2] Patey and Dyson, W. (1948) The Prognosis of Carcinoma of the Breast in Relation to the Type of Operation Performed, London.

[3] (2013) El Bolkainy, Pathology of Cancer.

[4] Omar, S., Khaled, H., Gaafar, R., et al. (2003) Breast Cancer in Egypt; a Review of 
Disease Presentation and Detection Strategies. La Revue de Santé de la Mediterranéeorientale, 9, 448-463.

[5] Mokhtar, N., Gouda, I. and Adel, I. (2007) Cancer Pathology Registry. NCI, Cairo.

[6] Clough, K.B., Kaufman, G.J. and Nos, C. (2010) Improving Breast Cancer Surgery: A Classification and Quadrant per Quadrant Atlas for Oncoplastic Surgery. Annals of Surgical Oncology, 17, 1375-1391. 